\title{
The personal and the organisational perspective on iatrogenic harm: bridging the gap through reconciliation processes
}

\author{
Graham P Martin, ${ }^{1}$ Sarah Chew, ${ }^{1}$ Thomas R Palser ${ }^{1,2}$
}

\begin{abstract}
'Department of Health Sciences, Centre for Medicine, University of Leicester, SAPPHIRE Group, Leicester, UK

${ }^{2}$ Department of Upper Gastrointestinal Surgery, Leicester Royal Infirmary, University Hospitals of Leicester, Leicester, UK
\end{abstract}

\section{Correspondence to} Dr Graham P Martin, Department of Health Sciences, Centre for Medicine, University of Leicester, SAPPHIRE Group, University Road, Leicester LE1 7RH, UK; gpm7@le.ac.uk

Accepted 17 May 2017 Published Online First 26 July 2017

\section{Linked}

- http://dx.doi.org/10.1136/ bmjgs-2016-005804

CrossMark

To cite: Martin GP, Chew S, Palser TR. BMJ Qual Saf 2017;26:779-781.
A pervasive theme of healthcare reform globally is greater candour about the imperfections of care quality, particularly for patients and family members when things go wrong. Numerous healthcare systems now have published policies around disclosure. However, as Moore and Mello document in their paper in this issue of BMJ Quality and Safety, ${ }^{1}$ details about how, what and when to disclose are scant, and based on minimal evidence about what works for patients, families, clinicians and organisations. Moore and Mello provide important insights from New Zealand, where a mandatory system for compensation following treatment injuries has been in place for over 40 years, on how to achieve reconciliation that satisfies the concerns of aggrieved patients and carers while being acceptable to clinicians and organisations.

Moore and Mello relate their findings in particular to the North American context. The traditional medical malpractice liability system in the USA has long been criticised for its cumbersome, lengthy procedures, financial risks to all parties (healthcare organisations, patients and families, and lawyers), and tendency to prioritise minimisation of liability over learning and improvement. ${ }^{2}$ Communication-and-resolution programmes have been offered as an approach that might meet the expectations of patients and families more swiftly and sensitively, and encourage learning from mistakes rather than suppression and concealment. ${ }^{3}$ In England, too, there have been moves towards emulating systems in place in other countries to facilitate early resolution of compensation claims and encourage learning from incidents, with calls for the introduction of a 'rapid resolution an redress system' for obstetric errors, ${ }^{4}$ and a shift in the focus of the NHS Litigation Authority, to be renamed NHS Resolution. ${ }^{5}$

More broadly, openness has been a major theme of recent policy reform in England. A culture of opacity and secrecy was strongly implicated in recent high-profile failings in the quality of care in Stafford and Morecambe Bay, ${ }^{67}$ where problems of quality and safety over prolonged periods persisted despite warning signs, ${ }^{8}$ and were associated with poor outcomes including unnecessary deaths. Responses to these failings have emphasised the importance of openness and transparency to learning and improvement. ${ }^{9-11}$ Long-standing concerns about the proclivity of the National Health Service to react to critical scrutiny in a defensive and obstructive manner-what Sir Robert Francis called its 'institutional instinct (towards) concealment' rather than embracing learning ${ }^{6}$ - have given rise to a host of policy interventions designed to foster openness. These include, most notably, a legal 'duty of candour' that compels all healthcare organisations to provide information, support and an apology to patients and families should harm be caused in the course of care. ${ }^{12}$ The duty of candour has been accompanied by a range of other policies with similar intent: a requirement that NHS organisations use complaints investigations to learn lessons and improve care ${ }^{13}$; greater support for staff who voice concerns about colleagues or systems; new requirements regarding the publication of data by NHS organisations; and a new regime of inspection by the Care Quality Commission (the 
regulator), alongside a much vaunted 'learning from mistakes league table,' which seeks to compare organisations on the basis primarily of staff's views of their culture around openness, learning and protection for 'whistleblowers."

Well-intentioned policies like these, however, can have unintended consequences. Interventions such as league tables can very quickly transform a concern with a problem into a concern with a metric-and result in a focus on appearances rather than a focus on improvement. ${ }^{15}{ }^{16}$ Some commentators have expressed similar concerns that a statutorily enforced duty of candour, encompassing not just disclosure of what went wrong but also an obligatory apology, might produce contrived, formulaic acts that prioritise satisfying legal requirements over reconciling aggrieved parties. ${ }^{17} 18$ Indeed, guidance for professionals on how to enact the duty of candour is at pains to clarify the distinction between 'saying sorry' and 'admitting legal liability, ${ }^{19}$ personal or organisational. Yet such approaches may fail to attend to the main objective of honesty and reconciliation from patients and families' point of view, or even create a process that does more harm than good. Contrary to what might be assumed, obtaining compensation is often not the dominant motivation for patients or families who complain about the quality of care; preventing recurrence, making staff aware of the impact of actions and obtaining an explanation are often more prominent concerns. ${ }^{20} 21$ Allied to these findings, other studies have indicated a gap between what patients and healthcare staff expect from complaints-resolution systems ('the expectations gap'), ${ }^{22} 23$ with patients who hope for remedial work to reduce risk to others often left disappointed. ${ }^{22}$ Unless their expectations are deliberately taken into account, patients may continue to be left dissatisfied by reconciliation processes.

In this light, perhaps the most striking and pervasive theme in Moore and Mello's paper is the value placed by patients and families on meaningful efforts by staff to understand the experience and impact of patient safety incidents from their perspective. Persistent and powerful in the accounts of the participants is the importance placed on an acknowledgement of the impact of the incident-which may be long-lasting, profound and indirect. Much of it may fall well outside the frame of reference of a biomedical or organisational understanding of the consequences of the incident. To give one example: an incident involving a participant's hearing was, for the doctor, an unfortunate but ultimately moderate accident; for the participant, a musician, it was a major, life-altering event. ${ }^{1}$ As a recent UK government-sponsored review noted, organisationally defined thresholds of harm may serve a helpful analytical purpose, but 'terms such as 'low' or 'moderate' harm (can be) unhelpful as a way of structuring a conversation with a patient or their carer. ${ }^{11}$
There is thus sometimes a chasm between the perspective of the system and the perspective of patients and families; for families, it is important to bridge this chasm. Patients and families may well want the clinician and organisation to learn from errors, but they also need them to recognise and understand the consequences of those errors. Professional guidance advocates an apology that is 'personalised,' rather than framed as 'a general expression of regret about the incident on the organisation's behalf. ${ }^{19}$ Moore and Mello's findings suggest this is important but not sufficient: what is needed is recognition that what an organisation sees as a lapse (or a process fallibility, a gap in training or an opportunity for learning) can look quite different from the victim's standpoint, and a sincere attempt to understand that standpoint.

Taking on such a different standpoint can be difficult in itself. The difficulty may be compounded by an environment in which calls for openness are permeated by concerns that, while well meaning, are essentially instrumentalist and organisationally oriented: legal compliance; supporting staff to acknowledge and report mistakes; using learning from incidents to improve. The challenge is both creating guidance for clinicians that incorporate the importance of acknowledgement and empathy for the perspective of patients and families, and creating supportive organisational environments that allow this to flourish alongside the other system-oriented objectives for openness.

Acknowledgements Graham Martin acknowledges the support of the NIHR Collaboration for Leadership in Applied Health Research and Care East Midlands (CLAHRC EM). The views expressed are those of the author(s) and not necessarily those of the NHS, the NIHR or the Department of Health.

Competing interests None declared.

Provenance and peer review Commissioned; internally peer reviewed.

(C) Article author(s) (or their employer(s) unless otherwise stated in the text of the article) 2017. All rights reserved. No commercial use is permitted unless otherwise expressly granted.

\section{REFERENCES}

1 Moore J, Mello MM. Improving reconciliation following medical injury: a qualitative study of responses to patient safety incidents in New Zealand. BMJ Qual Saf 2017;26:788-98.

2 Niel MBV, Shah R, DeVoe T, et al. Patient representation in communication and resolution programs: what is the best model? Healthcare Professional Liability Review 2016;1(1).

3 Mello MM, Boothman RC, McDonald T, et al. Communication-and-resolution programs: the challenges and lessons learned from six early adopters. Health Aff 2014;33:20-9.

4 National Maternity Review. 2016. Better births: improving outcomes of maternity services in England. London: NHS England.

5 Hansard. House of Commons vol. 623 col. 770. Mar 21, 2017. 
6 Mid Staffordshire NHS Foundation Trust Public Inquiry. Report of the Mid Staffordshire NHS Foundation Trust Public Inquiry. London: The Stationery Office, 2013.

7 Kirkup B. The report of the Morecambe Bay investigation. London: The Stationery Office, 2015.

8 Martin GP, McKee L, Dixon-Woods M. Beyond metrics? Utilizing 'soft intelligence' for healthcare quality and safety. Soc Sci Med 2015;142:19-26.

9 Department of Health. Hard truths: the journey to putting patients first. London: The Stationery Office, 2013.

10 National Advisory Group on the Safety of Patients in England. A promise to learn - a commitment to act: improving the safety of patients in England. London: Department of Health, 2013.

11 Dalton D, Williams N. Building a culture of candour: a review of the threshold for the duty of candour and of the incentives for care organisations to be candid. London: Royal College of Surgeons, 2014.

12 Care Quality Commission. 2015. Health and social care act 2008 (regulated activities) regulations 2014: Regulation 20: Care Quality Commission.

13 NHS England. 2016. NHS England Complaints Policy. Redditch: NHS England.

14 Care Quality Commission. 2017. The state of care in NHS acute hospitals: 2014 to 2016: Newcastle-Upon-Tyne: CQC.
15 Bevan G, Hood C. What's measured is what matters: targets and gaming in the English public health care system. Public Adm 2006;84:517-38.

16 Dixon-Woods M, Baker R, Charles K, et al. Culture and behaviour in the English National Health Service: overview of lessons from a large multimethod study. BMJ Qual Saf 2014;23:106-15.

17 Quick O. Regulating and legislating safety: the case for candour. BMJ Qual Saf 2014;23:614-8.

18 Hawkes N. Admitting when mistakes are made. BMJ 2015;351:h4474.

19 Nursing and Midwifery Council, General Medical Council. Openness and honesty when things go wrong: the professional duty of candour. London: GMC, 2015.

20 Bark P, Vincent C, Jones A, et al. Clinical complaints: a means of improving quality of care. Qual Health Care 1994;3:123-32.

21 Bismark M, Dauer E, Paterson R, et al. Accountability sought by patients following adverse events from medical care: the New Zealand experience. CMAJ 2006;175:889-94.

22 Bismark MM, Spittal MJ, Gogos AJ, et al. Remedies sought and obtained in healthcare complaints. BMJ Qual Saf 2011;20:806-10.

23 Friele RD, Reitsma PM, de Jong JD. Complaint handling in healthcare: expectation gaps between physicians and the public; results of a survey study. BMC Res Notes 2015;8:529. 